\title{
BMJ Open Excess costs of alcohol-dependent patients in German psychiatric care compared with matched non-alcohol- dependent individuals from the general population: a secondary analysis of two datasets
}

Judith Dams, ${ }^{1}$ Angela Buchholz, ${ }^{2}$ Ludwig Kraus, ${ }^{3,4,5}$ Jens Reimer, ${ }^{6,7}$ Norbert Scherbaum, ${ }^{8}$ Alexander Konnopka, ${ }^{1}$ Hans-Helmut König ${ }^{1}$

To cite: Dams J, Buchholz A, Kraus L, et al. Excess costs of alcohol-dependent patients in German psychiatric care compared with matched nonalcohol-dependent individuals from the general population: a secondary analysis of two datasets. BMJ Open 2018;8:e020563. doi:10.1136/ bmjopen-2017-020563

- Prepublication history and additional material for this paper are available online. To view these files, please visit the journal online (http://dx.doi org/10.1136/bmjopen-2017020563).

AK and $\mathrm{H}-\mathrm{HK}$ contributed equally.

Received 10 November 2017

Revised 27 June 2018

Accepted 27 July 2018

D Check for updates

(c) Author(s) (or their employer(s)) 2018. Re-use permitted under CC BY-NC. No commercial re-use. See rights and permissions. Published by BMJ.

For numbered affiliations see end of article.

Correspondence to

Dr Judith Dams; j.dams@uke.de

\section{ABSTRACT}

Objectives Heavy alcohol use can cause somatic and mental diseases, affects patients' social life and is associated with social isolation, unemployment and reduced quality of life. Therefore, societal costs of alcohol dependence are expected to be high. The aim of this study was to estimate excess costs of patients with alcohol dependence diagnosed using the Diagnostic and Statistical Manual of Mental Disorders, 4th Edition criteria compared with individuals without alcohol dependence in Germany.

Design In a secondary analysis, baseline data of patients with alcohol dependence enrolled in a randomised controlled trial (German Clinical Trials Register DRS00005035) were compared with data collected via a telephone survey from individuals without alcohol dependence and that had been matched by entropy balancing. Health service use was evaluated retrospectively for a 6-month period.

Settings Four German psychiatric university clinics (patients with alcohol dependence) and the German general adult population (individuals without alcohol dependence).

Participants $n=236$ adult patients with alcohol dependence and $n=4687$ adult individuals without alcohol dependence.

Primary and secondary outcome measures The excess costs of health service use, absenteeism and unemployment of patients with alcohol dependence were calculated and compared with individuals without alcohol dependence. In subgroup analyses, the associations between excess cost and gender, comorbidities and the duration of disease were investigated.

Results Total 6-month excess costs of $€ 11839$ (95\% Cl $€ 11529$ to $€ 12147)$ were caused by direct excess costs of $€ 4349(95 \% \mathrm{Cl} € 4129$ to $€ 4566)$ and indirect costs of $€ 7490$ (95\% Cl €5124 to €9856). In particular, costs of inpatient treatment, formal long-term care, absenteeism and unemployment were high.

Conclusions Alcohol dependence causes substantial direct and indirect excess costs. Cost-effective
Strengths and limitations of this study

- A new statistical approach was used to derive excess costs for alcohol dependence combining two data sets through entropy balancing to adjust for differences in sociodemographic and clinical characteristics.

- Missing values were managed through multiple imputation.

- The skewness of cost data was taken into account by applying generalised linear models with a gamma distribution and a log-link function.

- Some cost categories were not available, such as data on crime, accidents, medication costs and presentism.

- Recruitment took place in specialised psychiatric university clinics, thus costs due to inpatients psychiatric treatment may be overestimated.

interventions to prevent and treat alcohol dependence are urgently needed.

Trial registration number DRKS00005035.

\section{INTRODUCTION}

In 2010, German adults consumed on average $11.8 \mathrm{~L}$ of pure alcohol per year, with $16.8 \mathrm{~L}$ and $7.0 \mathrm{~L}$ consumed by men and women, respectively. ${ }^{1}$ Approximately $35 \%$ of the German population are estimated to have at least one episode of heavy drinking ( $\geq 5$ glasses of alcohol per day). ${ }^{23}$ Excessive use of alcohol is harmful to peoples' health and causes severe societal problems. The WHO estimated that $5.9 \%$ of all deaths result from the use of alcohol. ${ }^{4}$ In persons aged 20-39 years, $25 \%$ of deaths are caused by alcohol addiction. ${ }^{4}$ Alcohol-related diseases such as hepatitis, pancreatitis and various psychiatric 
disorders (eg, depression) cause a reduced quality of life. ${ }^{5}$ Negative social consequences of alcohol dependence (AD) include unemployment and social isolation.

According to the Diagnostic and Statistical Manual of Mental Disorders, 4th Edition criteria, ${ }^{7}$ persons are diagnosed with $\mathrm{AD}$ if at least three of the following criteria are fulfilled: tolerance; withdrawal symptoms or clinically defined alcohol withdrawal syndrome; use of larger amounts of alcohol for longer periods than intended; persistent desire or unsuccessful efforts to cut down on alcohol use; time is spent obtaining alcohol or recovering from effects; social, occupational and recreational pursuits are given up or reduced because of alcohol use; and use is continued despite knowledge of alcohol-related harm (physical or psychological). Overall, 6.5\% of the German population have been estimated to be alcohol dependent, corresponding to 3.4 million persons. ${ }^{38}$ Thus, the social and economic burden of $\mathrm{AD}$ is high. ${ }^{9}{ }^{10}$

The economic relevance of $\mathrm{AD}$ has been assessed by numerous studies. ${ }^{11-13}$ In general, total societal costs consist of direct and indirect costs. ${ }^{14}$ Direct costs refer to the monetary value of used resources (eg, hospital treatment, outpatient treatment or medication). Indirect costs represent the productivity loss due to morbidity or premature mortality. An international review of costof-illness studies summarised existing literature on the economic burden of $\mathrm{AD}$ and expressed its findings in US\$ purchasing power parities (US\$-PPP) (adjusted to the year 2006). ${ }^{11}$ Total societal costs were estimated to be between US\$-PPP 5228 million for Australia and US\$-PPP 216155 million for the USA. For Germany, total societal costs were estimated to be US\$-PPP 32540 million. Direct costs were estimated to be US\$-PPP 9421 million with US\$-PPP 3233 million due to inpatient treatment and US\$-PPP 6188 million due to outpatient treatment. A recent cohort study (analysing data draw from a large sample size of 606847 patients with $\mathrm{AD}$ from Catalonia) identified healthcare costs of $€ 1290$ per person per year. ${ }^{15}$ In this study, being male, more extensive alcohol consumption, tobacco use and lower socioeconomic status were associated with higher healthcare costs. As varying cost categories were used by the reviewed studies, results were difficult to compare.

Total societal costs of alcohol consumption have mostly been estimated using the so-called top-down approach, combining aggregated data and using the alcohol attributable fraction (AAF) approach to estimate the contribution of alcohol use disorder in cause-specific morbidity and mortality from different sources. ${ }^{16}{ }^{17}$ However, top-down studies cannot be used to analyse the influence of sociodemographic or clinical patient characteristics on costs. Furthermore, they are limited by the definition of $\mathrm{AAF}$ as the proportion of cases attributable to all alcohol consumption. As health economic researchers are often interested in identifying predictors of costs or detecting subgroups of patients with high costs, ${ }^{11}{ }^{12}$ bottom-up studies using patient-level data are needed. Moreover, when calculating the economic burden of a disease, costs are often overestimated due to the inclusion of comorbidities in the calculation of costs. To avoid this problem, excess costs representing the difference between costs of patients with a specific disease and (otherwise identical) individuals without this disease can be calculated, resulting in the calculation of costs solely associated with the disease itself. However, bottom-up studies often only collect data of patients with $\mathrm{AD}$, thus data of individuals without $\mathrm{AD}$ are not available. Our statistical approach overcomes this disadvantage by using entropy balancing to combine data of patients with $\mathrm{AD}$ and individuals without $\mathrm{AD}$. Our analyses may therefore assist in the use of data from existing bottom-up studies to derive excess costs.

Even though it is well known that the economic burden of $\mathrm{AD}$ is high, excess costs of $\mathrm{AD}$ were only estimated by one recently published bottom-up study considering the economic burden of AD in Germany. ${ }^{18}$ Total costs were reported to be $50 \%$ higher among patients with $\mathrm{AD}$ compared with individuals without. Calculations of indirect costs in particular differed to results of previous top-down studies. Consequently, the authors called for further cost-of-illness studies, using a bottom-up approach, in order to compare results with widely used top-down studies. Therefore, the aim of this study was to estimate excess costs of patients with $\mathrm{AD}$ in Germany using a bottom-up approach.

\section{METHODS}

Excess costs were calculated by comparing costs of individuals with and without $\mathrm{AD}$, adjusting for sociodemographic characteristics and comorbidities. The cost calculation of patients with $\mathrm{AD}$ was based on the baseline assessment of patients enrolled in a multicentre clinical trial. Cost calculation of the comparison group of individuals without $\mathrm{AD}$ was based on data collected via a nationally representative telephone survey.

\section{Study population with $A D$ in inpatient withdrawal treatment}

Data on patients with $\mathrm{AD}$ were taken from baseline interviews of patients included in a randomised clinical trial (Measurements in the Addictions for Triage and Evaluations - Levels of Care (MATE-LOC)) evaluating the effect of assessment-based recommendations for referral to subsequent treatment. Assessment of healthcare utilisation at baseline was retrospective for the preceding 6-month period. The trial was registered by the German Clinical Trials Register (DRS00005035). Data were collected between June 2013 and August 2014 in specialised alcohol withdrawal treatment units in four German psychiatric university clinics (Essen, Freiburg, Hamburg and Muenster). University clinics in Germany are maximum care hospitals in Germany financed by the public healthcare system and offer a wild range of treatment options. Psychiatric clinics participating in the MATE-LOC trial were specialised in detoxification and inpatient withdrawal treatment for AD. Patients 
with a primary diagnosis of $\mathrm{AD}$ were eligible to take part in the study. Patients were diagnosed by the attending psychologists/psychotherapists or psychiatrists/neurologist of the patients previous to and independent of the study inclusion. Only patients with $\mathrm{AD}$ and willing to have a withdrawal treatment were recommended for the MATE-LOC trial. Exclusion criteria included treatment for reasons other than $\mathrm{AD}$, severe cognitive impairment and psychotic disorder. Furthermore, participants were required to have German language skills and be literate. Even though patients were not selected by the severity of $\mathrm{AD}$, included patients were expected to be more strongly affected by $\mathrm{AD}$ than the average German patient with $\mathrm{AD}$ (see discussion section).

Baseline assessment included a short questionnaire on sociodemographic and clinical data and a measure of addiction severity, the severity of mental and somatic comorbid disorders and the level of functioning. The addiction severity was assessed using the Measurements in the Addictions for Triage and Evaluations questionnaire, ${ }^{19}$ which is a validated instrument assessing characteristics of people with drug and/or alcohol problems for triage and evaluation in treatment. It is conceptually constructed according to the International Classification of Diseases, the International Classification of Functioning, Disability and Health and World Health Organization classification system ${ }^{20}$ and includes consideration of the lifetime duration of heavy alcohol use. A total of 299 alcohol-dependent patients were enrolled; data from 250 participants were included in the analysis of the RCT. After exclusion of patients with cost outliers, 236 participants remained in the data set. Detailed description of the study protocol can be found elsewhere. ${ }^{21}$

\section{Study population without AD}

Individuals without $\mathrm{AD}$ were taken from a representative telephone survey of the German adult population. Five thousand and five adults ( $\geq 18$ years) were interviewed between March and April 2014. One hundred and forty participants were excluded due to missing values, as well as a further 35 participants due to being outliers in total costs. Furthermore, only participants who did not self-report an addiction disorder were considered. A total of 4687 individuals were included in the study as individuals without AD. Further information on the study design and results of healthcare utilisation have been published elsewhere. ${ }^{22}$

\section{Patient and public involvement}

The current study constitutes a secondary analysis of two datasets. Therefore, patients and the public were not directly involved in the development of the study protocol, including design, recruitment of patients and conduction of the study. Results will be available for participants through the journal publication.

\section{Health service use and costs}

We combined baseline trial data on healthcare utilisation of patients with $\mathrm{AD}$ included in the MATE-LOC trial with data of individuals without $\mathrm{AD}$ collected in the representative telephone survey of the German adult population. Differences in costs between patients with $\mathrm{AD}$ of the MATE-LOC trial and matched individuals without AD of the retrospective telephone survey are called 'excesscosts', as they represent costs that were solely due to $\mathrm{AD}$ and independent of further diseases and treatment choices.

In both groups, healthcare utilisation and sickness absence days in the preceding 6 months were assessed retrospectively. A modified version of the Client Socio-demographic and Service Receipt Inventory ${ }^{23}$ was used to evaluate direct and indirect costs from the societal perspective. Direct costs refer to the monetary value of used resources (eg, hospital treatment, outpatient treatment or medication), whereas indirect costs represent the productivity loss due to disease-related absence from work. ${ }^{24}$ Resource use and productivity losses were monetarily valued using German unit costs (online supplementary table S1).$^{25} 26$ If unit costs were not available for 2014 , they were adapted to the year 2014 by using the German consumer price index. ${ }^{27}$

Only costs available in both data sets were included. Direct costs included costs for outpatient physician and non-physician services (eg, occupational therapy, physiotherapy, logopaedics, sports therapy and alcohol-specific counselling) as well as formal and informal care. Formal care included care delivered by outpatient nursing services as well as professional household help. Informal care included care provided by family members and friends. Inpatient costs included stays in general hospitals, psychiatric hospitals or rehabilitation hospitals. Unfortunately, data on medication costs were not available for individuals without $\mathrm{AD}$ assessed by the telephone survey, because a serious recall bias for medication intake surveyed by telephone was expected. Therefore, it was not possible to calculate medication excess costs. Indirect costs included absenteeism and unemployment. The human capital approach was used to monetarily value time absent from work using full-time and part-time labour costs for manufacturing and service sectors. ${ }^{26} 28$

\section{Statistical analysis}

We used a statistical approach to estimate excess costs for patients with $\mathrm{AD}$ compared with individuals without $\mathrm{AD}$ that consisted of three steps: (1) imputing missing values in the MATE-LOC patient data set, (2) combining the data sets and (3) estimating excess costs using regression analyses.

\section{Imputation of missing values}

Imputation of missing values in data sets is recommended when the missing rate is above $5 \%-10 \% .{ }^{29}$ As the maximum missing rate per variable in the data set of individuals without $\mathrm{AD}$ was $0.8 \%$, no missing values were replaced and only complete cases were used. In contrast, the missing rate per variable in the data set of the MATE-LOC trial ranged between $0.0 \%$ and $34.8 \%$. 
Therefore, we decided to impute missing values using Multiple Imputation by Chained Equations (MICE)..$^{30} 31$ As imputation method predictive mean matching with 50 imputations was used. . $^{32-34}$

\section{Combining data sets}

Data on sociodemographic and clinical characteristics (age, sex, living situation, education and comorbidities) and healthcare utilisation, as well as productivity loss, were extracted from the data sets of MATE-LOC and the telephone survey. As both data sets were collected independently, the sociodemographic and clinical characteristics of participants with and without $\mathrm{AD}$ were different. To estimate excess cost solely caused by $\mathrm{AD}$, differences in sociodemographic and clinical characteristics were balanced using entropy balancing. ${ }^{35}$ The imputed MATE-LOC data set was used as a reference and remained unchanged. Data of the telephone survey were balanced for each of the 50 imputed MATE-LOC data sets, in order to ensure similar means and SEs of sociodemographic and clinical characteristics.

\section{Estimation of excess costs}

Excess costs were analysed using weighted regression analyses with costs as the dependent variable and the presence of $\mathrm{AD}$ as the independent variable. Weights derived by entropy balancing were included to adjust for differences in sociodemographic and clinical characteristics. In particular, two-part models (TPM) ${ }^{36}$ and generalised linear models (GLMs) with a gamma distribution and a log-link function ${ }^{37}$ were applied to account for skewed cost distributions. TPMs were used for analyses of cost categories with a substantial share of zero values (costs of outpatient non-physician treatment, costs of hospital treatment and indirect costs). GLMs were used for cost categories for which almost every participant incurred costs (costs of outpatient physician treatment, direct costs and total costs).

To minimise the impact of cost outliers, we excluded participants above the 99th percentile of total costs $(n=49$ : $\mathrm{n}=14$ for patients with $\mathrm{AD}$ and $\mathrm{n}=35$ for individuals without $\mathrm{AD})$. In an additional analysis, we winsorised costs of participants with total costs above the 99th percentile instead.

Subgroup analyses were carried out by gender and $\mathrm{AD}$ duration (short $\leq 7$ years $<$ medium $\leq 16$ years $<$ long). Furthermore, costs of patients without any comorbidity were calculated to determine the impact of psychological and somatic comorbidities.

Statistical analyses were conducted with R (V.3.4.1) and STATA V.15.1. The R-package 'mice' was used for multiple imputation. ${ }^{31}$ Entropy balancing was performed with the R-package 'ebal'. ${ }^{38}$ The STATA module 'twopm' was applied to compute TPMs. ${ }^{36}$

\section{RESULTS}

Table 1 presents sociodemographic and clinical characteristics of participants with and without AD before and after entropy balancing. Prebalancing, participants with and without $\mathrm{AD}$ differed in sociodemographic and
Table 1 Demographics and clinical sample characteristics prebalancing and postbalancing

\begin{tabular}{|c|c|c|c|}
\hline & $\begin{array}{l}\text { Patients } \\
\text { with AD } \\
\end{array}$ & $\begin{array}{l}\text { Individuals } \\
\text { without AD } \\
\text { prebalancing } \\
\end{array}$ & $\begin{array}{l}\text { Individuals } \\
\text { without AD } \\
\text { postbalancing }\end{array}$ \\
\hline & $(n=236)$ & $(n=4687)$ & $(n=4687)$ \\
\hline Covariates & Mean & Mean & Mean \\
\hline Age (mean) & 45.03 & 54.81 & 43.73 \\
\hline Gender (female \%) & 37 & 53 & 37 \\
\hline Person living not alone (\%) & 41 & 62 & 41 \\
\hline Unemployment (\%) & 26 & 4 & 8 \\
\hline \multicolumn{4}{|l|}{ Marital status (\%) } \\
\hline Unmarried & 49 & 27 & 49 \\
\hline Married & 17 & 43 & 17 \\
\hline Separated & 8 & 3 & 8 \\
\hline Divorced & 3 & 15 & 3 \\
\hline \multicolumn{4}{|l|}{ Educational level (\%) } \\
\hline None & 3 & 0 & 3 \\
\hline Vocational training & 30 & 27 & 30 \\
\hline Professional school & 33 & 33 & 33 \\
\hline A-level exam & 22 & 14 & 22 \\
\hline University & 12 & 25 & 12 \\
\hline \multicolumn{4}{|l|}{ Physical comorbidities (\%) } \\
\hline Lung disease & 8 & 16 & 8 \\
\hline Joint disease & 6 & 19 & 6 \\
\hline Metabolic disease & 9 & 26 & 9 \\
\hline Diabetes & 4 & 11 & 4 \\
\hline Chronic pain & 6 & 30 & 6 \\
\hline Gastrointestinal disease & 19 & 19 & 19 \\
\hline Cancer & 1 & 9 & 1 \\
\hline Cardiovascular disease & 22 & 34 & 22 \\
\hline Skin disease & 3 & 14 & 3 \\
\hline Osteoporosis & 1 & 8 & 1 \\
\hline \multicolumn{4}{|c|}{ Psychiatric/neurological comorbidities (\%) } \\
\hline Mental disorders & 36 & 10 & 29 \\
\hline Neurological disease & 8 & 1 & 8 \\
\hline
\end{tabular}

$A D$, alcohol dependence.

clinical characteristics. Patients with $\mathrm{AD}$ had a mean age of 45 years, while those without $\mathrm{AD}$ had a mean age of 54.8 years. Of patients with $\mathrm{AD}, 37 \%$ were women, whereas $53 \%$ of individuals without $\mathrm{AD}$ were female. Forty-nine per cent of the patients with $\mathrm{AD}$ were unmarried, whereas $27 \%$ of the individuals without $\mathrm{AD}$ were unmarried. Furthermore, patients with $\mathrm{AD}$ were less educated and had fewer somatic comorbidities but more mental and neurological diseases than individuals without AD. As expected, sociodemographic and clinical characteristics in both groups were similar after entropy balancing.

Costs were evaluated retrospectively for 6 months in 2014 in both datasets. Total costs per patient with AD were $€ 16378$ ( $\mathrm{SE} € 1060$ ), whereas total costs for individuals without $\mathrm{AD}$ were $€ 4539$ ( $\mathrm{SE} € 150$ ) (table 2). Thus, total excess costs of patients with $\mathrm{AD}$ compared with 
Table 2 Average 6-month costs and excess costs in euros of participants with and without AD in 2014

\begin{tabular}{|c|c|c|c|c|c|}
\hline & \multirow{2}{*}{$\begin{array}{l}A D \\
(n=236)\end{array}$} & \multirow{2}{*}{$\begin{array}{l}\text { Without AD } \\
(n=4687)\end{array}$} & \multicolumn{3}{|c|}{ Differences } \\
\hline & & & \multirow[b]{2}{*}{ Excess } & \multirow[b]{2}{*}{$95 \% \mathrm{Cl}$} & \multirow[b]{2}{*}{$P$ values } \\
\hline & Mean (SE) & Mean (SE) & & & \\
\hline \multicolumn{6}{|l|}{ Direct costs } \\
\hline \multicolumn{6}{|l|}{ Outpatient sector } \\
\hline Psychologist/psychotherapist & $100(26)$ & $45(3)$ & 55 & 0 to 110 & 0.050 \\
\hline Psychiatrist/neurologist & $46(12)$ & $22(1)$ & 24 & -2 to 50 & 0.075 \\
\hline Other physicians ${ }^{*}$ & $195(17)$ & $175(3)$ & 20 & 12 to 27 & $<0.001$ \\
\hline Non-physician specialists & $375(73)$ & $65(3)$ & 310 & 163 to 458 & $<0.001$ \\
\hline \multicolumn{6}{|l|}{ Inpatient sector } \\
\hline General hospital & $616(140)$ & $278(21)$ & 338 & -29 to 703 & 0.071 \\
\hline Psychiatric hospital & $2129(317)$ & $69(15)$ & 2060 & 1415 to 2704 & $<0.001$ \\
\hline Rehabilitation hospital & $579(173)$ & $74(9)$ & 505 & 139 to 871 & 0.007 \\
\hline \multicolumn{6}{|l|}{ Home care sector } \\
\hline Formal care & $1184(403)$ & $73(11)$ & 1111 & 318 to 1904 & 0.006 \\
\hline Informal care & $56(16)$ & $130(11)$ & -74 & -155 to 7 & 0.073 \\
\hline Total direct costs* & $5280(568)$ & $931(44)$ & 4349 & 4129 to 4566 & 0.000 \\
\hline \multicolumn{6}{|l|}{ Indirect costs } \\
\hline Absenteeism & 2084 (326) & $753(36)$ & 1331 & 623 to 2040 & $<0.001$ \\
\hline Unemployment & 9014 (997) & 2855 (139) & 6159 & 5121 to 7196 & $<0.001$ \\
\hline Total indirect costs & 11098 (968) & 3608 (140) & 7490 & 5124 to 9856 & $<0.001$ \\
\hline Total cost ${ }^{\star}$ & $16378(1.060)$ & 4539 (150) & 11839 & 11529 to 12147 & $<0.001$ \\
\hline
\end{tabular}

*Estimated with generalised linear models (GLM); the rest was estimated with two-part models .

$A D$, alcohol dependence.

individuals without were $€ 11839$ (95\% CI $€ 11529$ to $€ 12$ 147). Direct excess costs were $€ 4349$ (95\% CI $€ 4129$ to $€ 4566$ ) for patients with $\mathrm{AD}$ and were mainly caused by excess costs of inpatient treatment in general, psychiatric and rehabilitation hospitals, as well as formal care. Indirect excess costs of patients with $\mathrm{AD}$ amounted to $€ 7490$ (95\% CI $€ 5124$ to $€ 9856)$. In summary, all cost categories were higher for patients with $\mathrm{AD}$ than for individuals without $\mathrm{AD}$, except for costs due to informal care $(€-74$; $95 \% \mathrm{CI} €-155$ to $€ 7)$. All differences between costs for patients with $\mathrm{AD}$ and individuals without $\mathrm{AD}$ were statistically significant, with the exceptions of outpatient treatment by psychologists/psychotherapist, psychiatrist/ neurologist, inpatient treatment in general hospitals and informal care.

Results of analyses stratified by the lifetime duration of heavy alcohol use are presented in table 3 and table 4 . The analyses revealed differences in direct excess costs. Patients with a short lifetime duration of heavy alcohol use had direct excess costs of $€ 3504$ (95\% CI $€ 3101$ to $€ 3911)$ compared with individuals without $\mathrm{AD}$, whereas direct excess costs for patients with a long lifetime duration of heavy alcohol use were $€ 5925$ (95\% CI $€ 5448$ to $€ 6403$ ). Indirect excess costs of patients with a short, medium or long lifetime duration of heavy alcohol use were $€ 7571$ (95\% CI $€ 4206$ to $€ 10$ 935), $€ 6786$ (95\%
CI $€ 3183$ to $€ 10389)$ and $€ 7902 \quad(95 \%$ CI $€ 3876$ to $€ 11929)$, respectively.

Results of analyses by gender are presented as online supplementary table S2. Direct excess costs were $€ 4284$ (95\% CI $€ 3873$ to $€ 7247)$ for women with AD compared with women without $\mathrm{AD}$ and $€ 4165$ (95\% CI $€ 3862$ to $€ 4472)$ for men with $\mathrm{AD}$ compared with men without $\mathrm{AD}$. Women had higher direct excess costs for inpatient treatment in psychiatric hospitals and rehabilitation, whereas men had higher direct excess costs for formal care. Furthermore, indirect excess costs for men with AD were $€ 7164$ (95\% CI $€ 4203$ to $€ 10$ 127) compared with men without $\mathrm{AD}$, whereas indirect excess costs for women with AD were $€ 6621$ (95\% CI €2635 to €10 607) compared with women without AD.

Excess costs of participants without mental or somatic comorbidities are shown in the online supplementary table S3. Patients with $\mathrm{AD}$ and without any comorbidity had direct excess costs of $€ 2836$ ( $95 \%$ CI $€ 1340$ to $€ 4333$ ) when compared with healthy individuals. Indirect excess costs were $€ 9103$ (95\% CI $€ 5360$ to $€ 12847$ ) for patients with $\mathrm{AD}$ and without mental or somatic comorbidities, compared with healthy individuals.

Sensitivity analyses on outliers resulted in only small changes. Compared with the main analysis, excess costs were higher in the subgroup with winsorised cost outliers 
Table 3 Average 6-month costs in euros of participant with and without AD in 2014 for different lifetime duration of heavy alcohol use

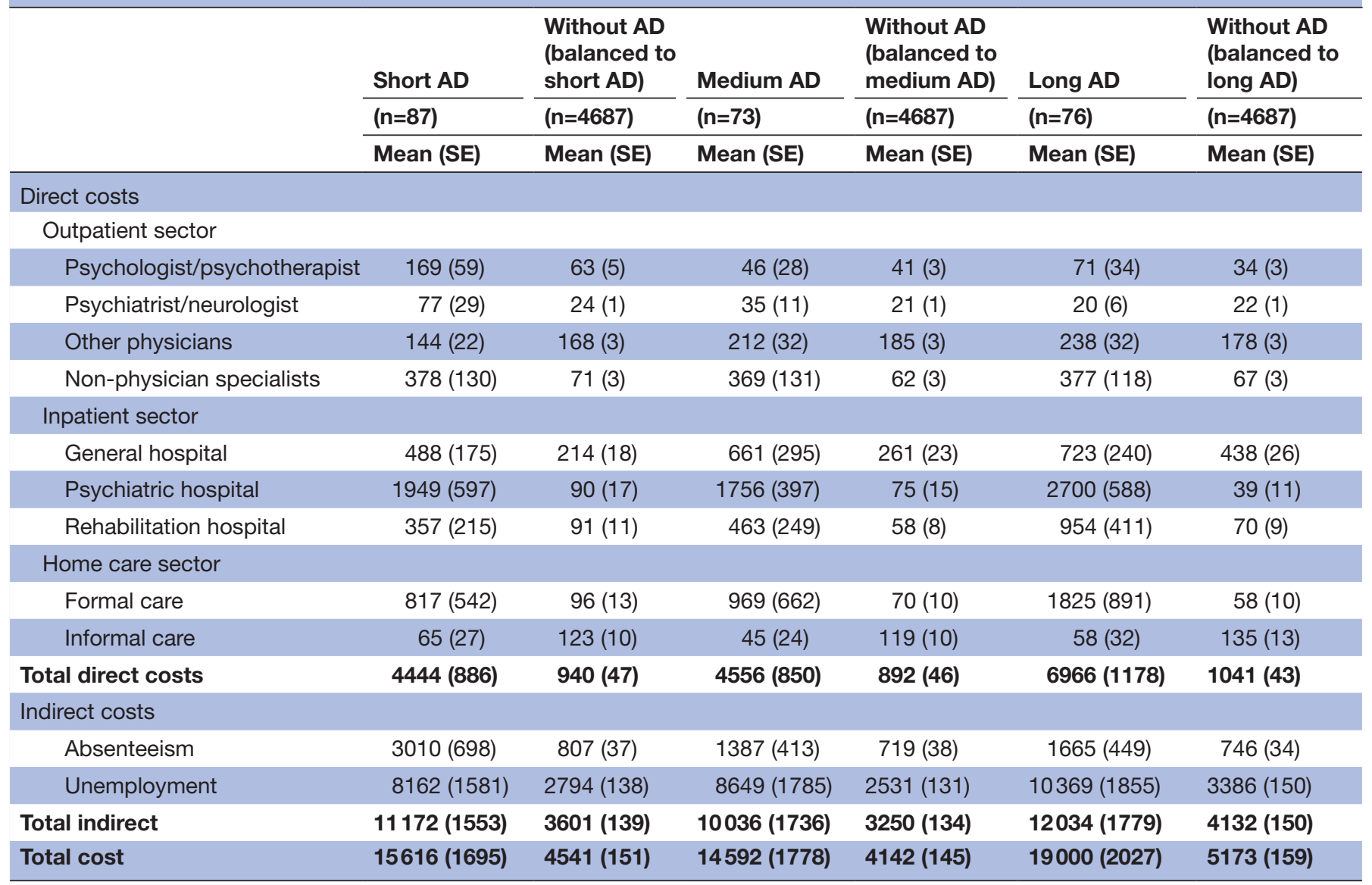

*Short $\leq 7$ years $<$ medium $\leq 16$ years $<$ long.

$A D$, alcohol dependence.

where total excess costs and direct excess costs of patients with $\mathrm{AD}$ were $€ 11171$ (95\% CI $€ 6599$ to $€ 11430)$ and $€ 5066$ (95\% CI $€ 2537$ to $€ 5250)$ compared with individuals without $\mathrm{AD}$. Indirect excess costs of $€ 7152(95 \%$ CI $€ 3071$ to $€ 11233$ )) were almost equal to those of the main analysis.

For all subgroup analyses, differences between patients with $\mathrm{AD}$ and individuals without $\mathrm{AD}$ in total costs, as well as direct and indirect costs, remained statistically significant, except for the total excess costs of patients without any comorbidity.

\section{DISCUSSION}

Our analysis revealed a high economic burden of $\mathrm{AD}$ with 6-month total excess costs of $€ 11839$ for patients with AD compared with individuals without. Indeed, costs were almost four times higher for patients with $\mathrm{AD}$ compared with individuals without $\mathrm{AD}$.

Approximately 2/3 of the total excess costs were due to indirect excess costs, with $82 \%$ of the indirect excess costs caused by unemployment. When compared with results reported by the only other study on excess cost for patients with $\mathrm{AD}$ in Germany, ${ }^{18}$ indirect excess costs in our study were much higher (€7490 vs $€ 1051$ ), particularly costs due to unemployment (€6159 vs $€ 373$ ). In our analysis, the duration of unemployment was not specifically assessed and therefore assumed to be 6 months. ${ }^{39}$ The costs of unemployment may consequently be overestimated.

Unexpectedly, indirect costs decreased between a short and medium lifetime duration of heavy alcohol use. In subgroup analyses, patients with a short lifetime duration of heavy alcohol use showed higher indirect excess costs compared with patients with a medium lifetime duration of heavy alcohol use, although the numbers of comorbidities increased. Fifty-five per cent of the patients with a short lifetime duration of heavy alcohol use were employed, whereas only $42 \%$ of the patients with a medium lifetime duration of heavy alcohol use were employed. As indirect costs are linked to the employment rate, the higher employment rate among patients with a short lifetime duration of heavy alcohol use is responsible for higher indirect excess costs.

Direct excess costs amounted to only one-third of total excess costs. Cost of inpatient treatment amounted to more than three quarters of direct excess costs. Less 
Table 4 Average 6-month excess costs in euros of participant with and without AD in 2014 for different lifetime duration of heavy alcohol use

\begin{tabular}{|c|c|c|c|c|c|c|c|c|c|}
\hline & \multicolumn{3}{|c|}{ Short AD } & \multicolumn{3}{|c|}{ Medium AD } & \multicolumn{3}{|l|}{ Long AD } \\
\hline & Excess & $95 \% \mathrm{Cl}$ & $P$ values & Excess & $95 \% \mathrm{Cl}$ & $P$ values & Excess & $95 \% \mathrm{CI}$ & $P$ values \\
\hline \multicolumn{10}{|l|}{ Direct costs } \\
\hline $\begin{array}{l}\text { Psychologist } \\
\text { /psychotherapist }\end{array}$ & 106 & -15 to 228 & 0.087 & 5 & -52 to 63 & 0.861 & 37 & -32 to 105 & 0.294 \\
\hline $\begin{array}{l}\text { Psychiatrist/ } \\
\text { neurologist }\end{array}$ & 53 & -6 to 112 & 0.077 & 14 & -12 to 39 & 0.229 & -2 & -19 to 14 & 0.803 \\
\hline Other physicians* & -24 & -32 to -17 & $<0.001$ & 27 & 21 to 34 & $<0.001$ & 60 & 48 to 73 & $<0.001$ \\
\hline $\begin{array}{l}\text { Non-physician } \\
\text { specialists }\end{array}$ & 307 & 45 to 569 & 0.021 & 307 & 50 to 563 & 0.019 & 310 & 75 to 545 & 0.010 \\
\hline \multicolumn{10}{|l|}{ Inpatient sector } \\
\hline General hospital & 274 & -222 to 770 & 0.278 & 400 & -256 to 1055 & 0.232 & 285 & -401 to 972 & 0.415 \\
\hline Formal care & 721 & -344 to 1785 & 0.185 & 899 & -395 to 2191 & 0.173 & 1767 & 29 to 3504 & 0.046 \\
\hline Informal care & -58 & -153 to 36 & 0.225 & -74 & -165 to 17 & 0.109 & -77 & -177 to 22 & 0.128 \\
\hline Total direct costs ${ }^{*}$ & 3504 & 3101 to 3911 & 0.000 & 3664 & 3209 to 4117 & $<0.001$ & 5925 & 5448 to 6403 & $<0.001$ \\
\hline \multicolumn{10}{|l|}{ Indirect costs } \\
\hline Absenteeism & 2203 & 794 to 3611 & 0.002 & 668 & -193 to 1528 & 0.128 & 919 & -7 to 1847 & 0.052 \\
\hline Unemployment & 5368 & 3804 to 6932 & $<0.001$ & 6118 & 4418 to 7819 & $<0.001$ & 6983 & 4421 to 8151 & $<0.001$ \\
\hline Total indirect & 7571 & 4206 to 10935 & $<0.001$ & 6786 & 3183 to 10389 & $<0.001$ & 7902 & 3876 to 11929 & $<0.001$ \\
\hline Total cost ${ }^{\star}$ & 11075 & 10513 to 11639 & $<0.001$ & 10450 & 9897 to 11001 & $<0.001$ & 13827 & 13051 to 14605 & $<0.001$ \\
\hline
\end{tabular}

Short $\leq 7$ years $<$ medium $\leq 16$ years $<$ long.

*Estimated with generalised linear models (GLM); the rest was estimated with two-part models.

$A D$, alcohol dependence.

than $10 \%$ of inpatient treatment costs were incurred in general hospitals, with the remaining costs incurred due to treatment in psychiatric and rehabilitation hospitals, where patients are likely to have received specific $A D$ treatment. Excess costs of inpatient treatment increased with lifetime duration of heavy alcohol use. Furthermore, excess costs of formal care were high, in particular among patients with a long lifetime duration of heavy alcohol use, whereas excess costs of informal care were rather low. This might be explained by the absence of a partner and social contacts and/or their inability to provide support. ${ }^{40}$ Even if patients were not living alone, difficulties in relationships existed, thus relatives might not be able to provide support. In the literature, social deficits and isolation were reported for any kind of alcoholism independent of the severity of disease. ${ }^{41}$ Therefore, patients with $\mathrm{AD}$ may be forced to use formal rather than informal care.

Direct excess costs found in our study (€4349) were higher than those reported for Germany in literature $(€ 1836) .{ }^{18}$ In particular, excess costs of inpatient treatment ( $€ 2903$ vs $€ 469)$ and, to a lesser extent, of outpatient treatment $(€ 409$ vs $€ 314)$ were higher in our sample. However, excess costs for the treatment by physicians other than psychologist/psychotherapists or psychiatrists/neurologists were lower in our study (€20 vs €120).
These differences could be explained by differences in the recruitment of samples, as our sample was recruited in psychiatric university hospitals, whereas Manthey et $a l^{18}$ recruited patients via general practitioners. Specifically, when compared with statistics of the federal statistical office on all patients with $\mathrm{AD}$ receiving inpatient treatment in Germany, patients of the MATE-LOC trial were on average 10 years younger and more often male $(48 \%$ vs $65 \%){ }^{42}$ These two factors are known to be associated with a more harmful consumption of alcohol. ${ }^{43}$ Furthermore, mean length of inpatient-stays of patients in the MATE-LOC trial was approximately 10 days longer compared with other $\mathrm{AD}$ patients. ${ }^{42}$ Thus, compared with other patients with $\mathrm{AD}$, costs might be overestimated.

Additional analyses revealed men having higher excess costs than women, which is in line with results of the literature. ${ }^{11-1318}$ Gender differences in excess costs in our results were caused by the differences in indirect costs due to absenteeism and unemployment $(€ 7164$ vs $€ 6621)$. Men were more often unemployed and, if employed, were more often absent from work. Direct costs were similar for both groups (€4165 vs $€ 4284$ ).

Patients with $\mathrm{AD}$ and without any comorbidity had slightly higher excess costs compared with patients included in the main analysis. As patients with $\mathrm{AD}$ and 
without any comorbidity were all male and costs for male patients with $\mathrm{AD}$ are known to be higher compared with female, ${ }^{43}$ cost differences could be explained by gender differences.

\section{Strengths and limitations}

Our analysis has several strengths including its statistical approach and the data sets used. We combined different statistical approaches to derive excess costs for participants with and without $\mathrm{AD}$ for the German healthcare system. We used two independent data sets, which were combined by entropy balancing to adjust for differences in sociodemographic and clinical characteristics. As randomised controlled trials often do not include healthy controls, it was necessary to match a second data set by entropy balancing, which only controls for observed parameter. To our knowledge, this was the first time this statistical approach was used to derive excess costs for AD. Missing values were managed using MICE, a powerful statistical approach, useful for instances where when missing values occur completely at random or depend on observed data. Furthermore, our statistical approach took the skewness of cost data into account by applying GLMs with a gamma distribution and a log-link function or TPMs. In subgroup analyses, we investigated the effect of different sociodemographic and clinical characteristics.

However, there are some limitations in our study. First, we did not include all cost categories usually assessed in cost-of-illness studies for $\mathrm{AD}$, because data on crime, accidents, medication costs and presentism due to disability and early retirement were not available in both data sets used. These costs categories are known to be increased for patients with $\mathrm{AD},{ }^{18} 4445$ thus excess costs may have been underestimated. Second, SEs especially for inpatient costs, were high. We conducted several analyses to avoid methodological bias. We tested for variations in the number of imputations, iterations and nearest neighbours, for influences of outliers and for the number of missing values. The results of complete case analysis were similar to those received by the main analysis. We came to the conclusion that between variations of imputation were caused by the data itself, because a few patients with $\mathrm{AD}$ had very high costs for inpatient treatment. However, it might be possible that missing values were not (completely) random. Third, recruitment took place in specialised psychiatric university clinics, thus cost due to inpatient psychiatric treatment may be overestimated. Fourth, individuals who participated in the telephone survey were included based on self-reported diagnoses. As no clinical diagnoses were made and individuals might have concealed an $\mathrm{AD}$, data of the telephone survey may include some individuals with an addiction disorder. As these individuals with $\mathrm{AD}$ are likely to have higher costs than those without $\mathrm{AD}$, this bias would lead to an underestimation of excess costs. Furthermore, excess costs might differ, because individuals were asked to report any addiction disorder and not specifically AD. Fifth, a bias in costs for absenteeism may have occurred. Self-reported absenteeism from work is likely to be concealed, ${ }^{46}{ }^{47}$ thus excess costs may have been underestimated.

\section{Conclusion}

Our analysis revealed significant excess costs due to AD. Indirect excess costs were high, particularly those due to unemployment. Furthermore, direct excess costs, especially for inpatient treatment and formal home care, were high. High inpatient costs may have been caused by the recruitment of patients in specialised psychiatric university clinics. Additional analyses revealed that excess costs were higher for men compared with women, and costs increased with lifetime duration of heavy alcohol use. In order to reduce the high costs of $\mathrm{AD}$, cost-effective interventions to prevent and treat $\mathrm{AD}$ are urgently needed.

\section{Author affiliations}

${ }^{1}$ Department of Health Economics and Health Services Research, Hamburg Center for Health Economics (HCHE), University Medical Center Hamburg-Eppendorf, Hamburg, Germany

${ }^{2}$ Department of Medical Psychology, University Medical Center Hamburg-Eppendorf, Hamburg, Germany

${ }^{3}$ IFT Institute for Therapeutic Research Munich, Munich, Germany

${ }^{4}$ Department for Public Health Sciences, Stockholm University, Stockholm, Sweden

${ }^{5}$ Institute of Psychology, ELTE Eötvös Loránd University, Budapest, Hungary

${ }^{6}$ Centre for Interdisciplinary Addiction Research, University of Hamburg, Hamburg,

Germany

${ }^{7}$ Centre for Psychosocial Medicine, Health North, Bremen, Germany

${ }^{8}$ LVR-Hospital Essen, Department of Addictive Behavior and Addiction Medicine,

Medical Faculty, University of Duisburg-Essen, Essen, Germany

Acknowledgements We would like to thank Thomas Grochtdreis and Eleanor Quirke for their helpful comments on an earlier version of the manuscript. We would like to thank all involved patients and therapists for their participation.

Contributors AB, LK, JR, NS, AK and H-HK: conceived the study and developed the design. JD was responsible for the analysis and wrote the first draft. All authors contributed to its revision and final approval.

Funding This study was supported by the German Ministry of Education and Research grant numbers $01 \mathrm{GY} 1114$ and 01EH1101B.

Competing interests None declared.

Patient consent Obtained.

Ethics approval The ethics committee of the local medical association in Hamburg, the Ethik-Kommission der Ärtzekammer Hamburg (Reference Number PV4325) and the ethics committee at each of the participating sites have approved the study protocol. Ethical approval was granted in accordance with the principles of the Declaration of Helsinki.

Provenance and peer review Not commissioned; externally peer reviewed.

Data sharing statement Data from patients cannot be accessed by anyone who is not part of the research team due to ethical and confidentiality concerns.

Open access This is an open access article distributed in accordance with the Creative Commons Attribution Non Commercial (CC BY-NC 4.0) license, which permits others to distribute, remix, adapt, build upon this work non-commercially, and license their derivative works on different terms, provided the original work is properly cited, appropriate credit is given, any changes made indicated, and the use is non-commercial. See: http://creativecommons.org/licenses/by-nc/4.0/.

\section{REFERENCES}

1. World Health Organization (WHO). Global status report on alcohol and health. 2014 http://www.who.int/substance_abuse/publications/ global_alcohol_report/msbgsruprofiles.pdf (accessed 02 Nov 2016).

2. Gomes de Matos E, Atzendorf J, Kraus L, et al. Substanzkonsum in der Allgemeinbevölkerung in Deutschland [Substance use in the German general population]. Sucht 2016;62:271-81. 
3. Pabst A, Kraus L, Gomes de Matos E, et al. Substanzkonsum und substanzbezogene Störungen in Deutschland im Jahr 2012 [Substance use and stubstance use disorders in Germany in 2012]. Sucht 2013:59:321-31.

4. World Health Organization (WHO). Alcohol consumption: levels and patterns. 2015 http://www.who.int/substance_abuse/publications/ global alcohol report/profiles/deu.pdf (accessed 17 Jun 2016).

5. Ugochukwu C, Bagot KS, Delaloye S, et al. The importance of quality of life in patients with alcohol abuse and dependence. Harv Rev Psychiatry 2013;21:1-17.

6. Levola J, Aalto M, Holopainen A, et al. Health-related quality of life in alcohol dependence: a systematic literature review with a specific focus on the role of depression and other psychopathology. Nord $J$ Psychiatry 2014;68:369-84.

7. American Psychiatric Association. Diagnostic and statistical manual of mental disorders: DSM-IV. Washington (DC): American Psychiatric Association, 1994.

8. Bundesministerium für Gesundheit. Alkohol: Situation in Deutschland. 2016 http://www.drogenbeauftragte.de/drogen-undsucht/alkohol/alkohol-situation-in-deutschland.html (accessed 02 Nov 2016).

9. Gastfriend DR. A pharmaceutical industry perspective on the economics of treatments for alcohol and opioid use disorders. Ann N Y Acad Sci 2014;1327:n/a-30.

10. Kraus L, Pabst A, Piontek D, et al. Temporal changes in alcoholrelated morbidity and mortality in Germany. Eur Addict Res 2015;21:262-72.

11. Mohapatra S, Patra J, Popova S, et al. Social cost of heavy drinking and alcohol dependence in high-income countries. Int $J$ Public Health 2010;55:149-57.

12. Konnopka A, König HH. Direct and indirect costs attributable to alcohol consumption in Germany. Pharmacoeconomics 2007;25:605-18.

13. Olesen J, Gustavsson A, Svensson M, et al. The economic cost of brain disorders in Europe. Eur J Neurol 2012;19:155-62.

14. Eisenberg JM. Clinical economics. A guide to the economic analysis of clinical practices. JAMA 1989;262:2879-86.

15. Miquel L, Rehm J, Shield KD, et al. Alcohol, tobacco and health care costs: a population-wide cohort study ( $n=606947$ patients) of current drinkers based on medical and administrative health records from Catalonia. Eur J Public Health 2018;28:674-80.

16. Rehm J, Shield K, Rehm M, et al. Alcohol consumption, alcohol dependence, and attributable burden of disease in Europe: potential gains from effective interventions for alcohol dependence. Toronto, Canada: Centre for Addiction and Mental Health, 2012.

17. World Health Organization (WHO). Alcohol. 2015 http://www.who.int/ mediacentre/factsheets/fs349/en/ (accessed 27th June 2017).

18. Manthey J, Laramée P, Parrott S, et al. Economic burden associated with alcohol dependence in a German primary care sample: a bottom-up study. BMC Public Health 2016;16:906

19. Schippers G, Broekman T, Buchholz A. MATE 2.1. Manual and Protocol. English Edition: W. M. Cox. Bêta Boeken: Nijmegen, 2011.

20. Schippers GM, Broekman TG, Buchholz A, et al. Measurements in the Addictions for Triage and Evaluation (MATE): an instrument based on the World Health Organization family of international classifications. Addiction 2010;105:862-71.

21. Buchholz A, Friedrichs A, Berner M, et al. Placement matching of alcohol-dependent patients based on a standardized intake assessment: rationale and design of a randomized controlled trial. BMC Psychiatry 2014;14:286.

22. Grupp $\mathrm{H}$, König $\mathrm{H}-\mathrm{H}$, Konnopka $\mathrm{A}$. Health care utilisation and costs in the general population in Germany. Health Policy 2016;120:159-69.

23. Chisholm D, Knapp MR, Knudsen HC, et al. Client sociodemographic and service receipt inventory--European version: development of an instrument for international research. EPSILON study 5. European psychiatric services: inputs linked to outcome domains and needs. Br J Psychiatry Suppl 2000:s28-33.
24. Schöffski O, Graf von der Schulenburg J-M. Die Berechnung von Kosten und Nutzen [Caculation of costs and utilities]. Gesundheitsökonomische Evaluationen [Health economic evaluations]. Berlin Heidelberg: Springer-Verlag, 2012:23-42.

25. Bock JO, Brettschneider C, Seidl H, et al. Calculation of standardised unit costs from a societal perspective for health economic evaluation. Gesundheitswesen 2015;77:53-61.

26. Grupp H, König HH, Konnopka A. Calculation of standardised unit costs for the economic evaluation of mental disorders. Gesundheitswesen 2017;79:48-57.

27. Statistisches Bundesamt [Federal Statistical Office]. Verbraucherpreisindizes für Deutschland [consumer price index for Germany. Wiesbaden (Germany): Statistisches Bundesamt [Federal Statistical Office], 2014.

28. Statistisches Bundesamt [Federal Statistical Office]. Statistisches Jahrbuch 2013 [Statistical Year Book 2013. Wiesbaden (Germany): Statistisches Bundesamt [Federal Statistical Office], 2013.

29. Dong Y, Peng CY. Principled missing data methods for researchers. Springerplus 2013;2:222.

30. Azur MJ, Stuart EA, Frangakis C, et al. Multiple imputation by chained equations: what is it and how does it work? Int J Methods Psychiatr Res 2011;20:40-9.

31. Buuren S, Groothuis-Oudshoorn K. mice : multivariate imputation by chained equations in R. J Stat Softw 2011;45:1-67.

32. Enders CK. Applied missing data analysis. Guilford Publications, 2011.

33. Little RJA. Missing-data adjustments in large surveys. Journal of Business \& Economic Statistics 1988;6:287-96.

34. Molenberghs G, Kenward M. Missing data in clinical studies: Wiley, 2007.

35. Hainmueller J. Entropy balancing for causal effects: a multivariate reweighting method to produce balanced samples in observational studies. Political Analysis 2012;20:25-46.

36. Belotti F, Deb P, Manning W, et al. towpm: two-part models. Stata Journal 2015:15:3-20.

37. Moran JL, Solomon PJ, Peisach AR, et al. New models for old questions: generalized linear models for cost prediction. J Eval Clin Pract 2007:13:381-9.

38. Hainmueller J. ebal: Entropy reweighting to create balanced samples. 2014 http://CRAN.R-project.org/package=ebal (accessed 15 Mar 2016).

39. van Asselt AD, Dirksen CD, Arntz A, et al. Difficulties in calculating productivity costs: work disability associated with borderline personality disorder. Value Health 2008;11:637-44.

40. Temple MT, Fillmore KM, Hartka E, et al. A meta-analysis of change in marital and employment status as predictors of alcoho consumption on a typical occasion. Br J Addict 1991;86:1269-81.

41. Akerlind I, Hörnquist JO. Loneliness and alcohol abuse: a review of evidences of an interplay. Soc Sci Med 1992;34:405-14.

42. Statistisches Bundesamt [Federal Statistical Office]. Statistisches Jahrbuch 2017 [statistical year book 2017]. Wiesbaden (Germany): Statistisches Bundesamt [Federal Statistical Office], 2017.

43. Robert Koch Institut (RKI). GEDA 2010: Einflussfaktoren auf die Gesundheit: Alkoholkonsum [Risk factors on health: alcohol consumption]. Berlin: Robert Koch Institut (RKI), 2010.

44. Hansen B R, Waddell G. Legal access to alcohol and criminality:2017.

45. Scarborough $\mathrm{P}$, Bhatnagar $\mathrm{P}$, Wickramasinghe KK, et al. The economic burden of ill health due to diet, physical inactivity, smoking, alcohol and obesity in the UK: an update to 2006-07 NHS costs. J Public Health 2011;33:527-35.

46. Grøvle L, Haugen AJ, Keller A, et al. Poor agreement found between self-report and a public registry on duration of sickness absence. $J$ Clin Epidemiol 2012;65:212-8.

47. Voss M, Stark S, Alfredsson L, et al. Comparisons of self-reported and register data on sickness absence among public employees in Sweden. Occup Environ Med 2008;65:61-7. 